\title{
X-ray Computed Tomography for Micro Porosity in AZ91D Alloy
}

\author{
Hoonmo Park and Kenji Miwa \\ Light Metals Solidification Processing Group, Institute for Structural and Engineering Materials, \\ National Institute of Advanced Industrial Science and Technology, Nagoya 463-8560, Japan
}

This research has been clarified the method to precisely analyze characteristics of micro porosity in AZ91D alloy using X-ray computed tomography(XRCT). For analyzing the micro porosity, we used the defect-free sample that had 50 and $100 \mu \mathrm{m}$ sized artificial holes. The defining of peak in the variation of gray level could be used for analyzing the porosity or compounds. Increasing of image conversion coefficient $a$, increased the sensitivity of reconstructed image and showed even the difference of matrix. The optimized slice width for measuring the actual dimensions of micro porosity, depends on the size of micro porosity. If the slice width was too thin to analyze only the porosity, even the matrix would be detected as the porosity and the results would include the serious error. The accuracy of data derived from XRCT depends on the slice width related with the amounts of collected data.

(Received May 27, 2003; Accepted September 2, 2003)

Keywords: X-ray computed tomography, micro porosity, image conversion coefficient, slice width, AZ91D alloy

\section{Introduction}

Magnesium alloys have received much attention as the light structural materials in the aerospace and automotive industries. And the developments of industry have demanded the improvements of their properties and microstructure control using new processes. For the improvements of their properties and microstructure control, the behavior of porosity with an order of micrometer and the inner structure must be researched.

The X-ray computed tomography (XRCT) is one of the useful nondestructive tools for investigating the inner structure of various materials. ${ }^{1,2)}$ This technique measures the transmitted intensity of X-rays passed through an object from all angles in a plane through the object, and backcalculates a map of the object's X-ray linear attenuation coefficient for small volume elements in the plane. X-ray linear attenuation coefficient is the ratio of X-ray source intensity to recorded intensity after passing through the material as shown in eq. (1). ${ }^{3)}$

$$
\text { Linear Attenuation }=\ln \left(I_{0} / I\right)
$$

$I_{0}$ : the X-ray source intensity

I: the recorded intensity

The reconstructed image based on this map of X-ray linear attenuation coefficient, shows the position, size, and composition of the objects in the field of view of the image intensifier. With this characteristics derived from XRCT data, engineers can perform a variety of analyses to arrive at quantitative measurements of parameters that are of economic value to improving the overall product cycle from prototype development to real products. ${ }^{4,5)}$ In the case of a high resolution XRCT, it is possible to characterize the inner structure of materials within an order of micrometer without any destructive sample preparation which is needed in typical metallography. Therefore, the application of XRCT for materials science is on an increasing trend.

However, the most problematic matter in the XRCT analysis is that the operating conditions such as X-ray tube, image intensifier, image processing system and scan mode, seriously affect on the resolution of the XRCT analysis and the analysis result. Also, because the characteristics of alloy system such as the secondary dendrite arm size, solute segregation, grain size and casting defects result in only one function of density, the correct analysis method is necessarily demanded. Especially, these problems become more intensified for the analysis of the porosity with an order of micrometer than the macro scale analysis with an order of millimeter. These problems may cause the serious error in the dimensions of micro porosity derived from XRCT analysis.

Therefore, in order to determine the actual size and distribution of the porosity with an order of micrometer in magnesium alloys, it is very important to use the optimized operating conditions and the proper analysis method which consider the purpose of analysis and the characteristics of material.

This research was focused on the optimized operating conditions and the proper analysis method that could precisely analyze the characteristics of micro porosity in AZ91D alloy (Mg-9 mass\%Al-1 mass\%Zn). AZ91D alloy is a representative commercial magnesium alloy and its matrix structure strongly affects on the XRCT results. In this study, we investigated the effect of the image conversion coefficient $a$ and the slice width on the reconstructed image and the dimension of micro porosity derived from XRCT data in AZ91D alloy. Also the relation of slice width with the size of micro porosity was investigated. The image conversion coefficient $a$ is used as an important factor which is directly related with X-ray linear attenuation coefficient and can reflect the purpose of analysis and the characteristics of materials on the XRCT analysis results. And the slice width is proportional to the amounts of collected XRCT signal and affects the quality of reconstructed image. Therefore, the image conversion coefficient $a$ and slice width could be very important values in XRCT analysis for the dimensions of micro porosity in AZ91D alloy.

\section{Experimental Procedure}

In order to analyze the micro porosity in AZ91D alloy, the specimens have no porosity except for the artificial hole. The defect-free specimens with $6 \mathrm{~mm}$ in diameter and $10 \mathrm{~mm}$ in 

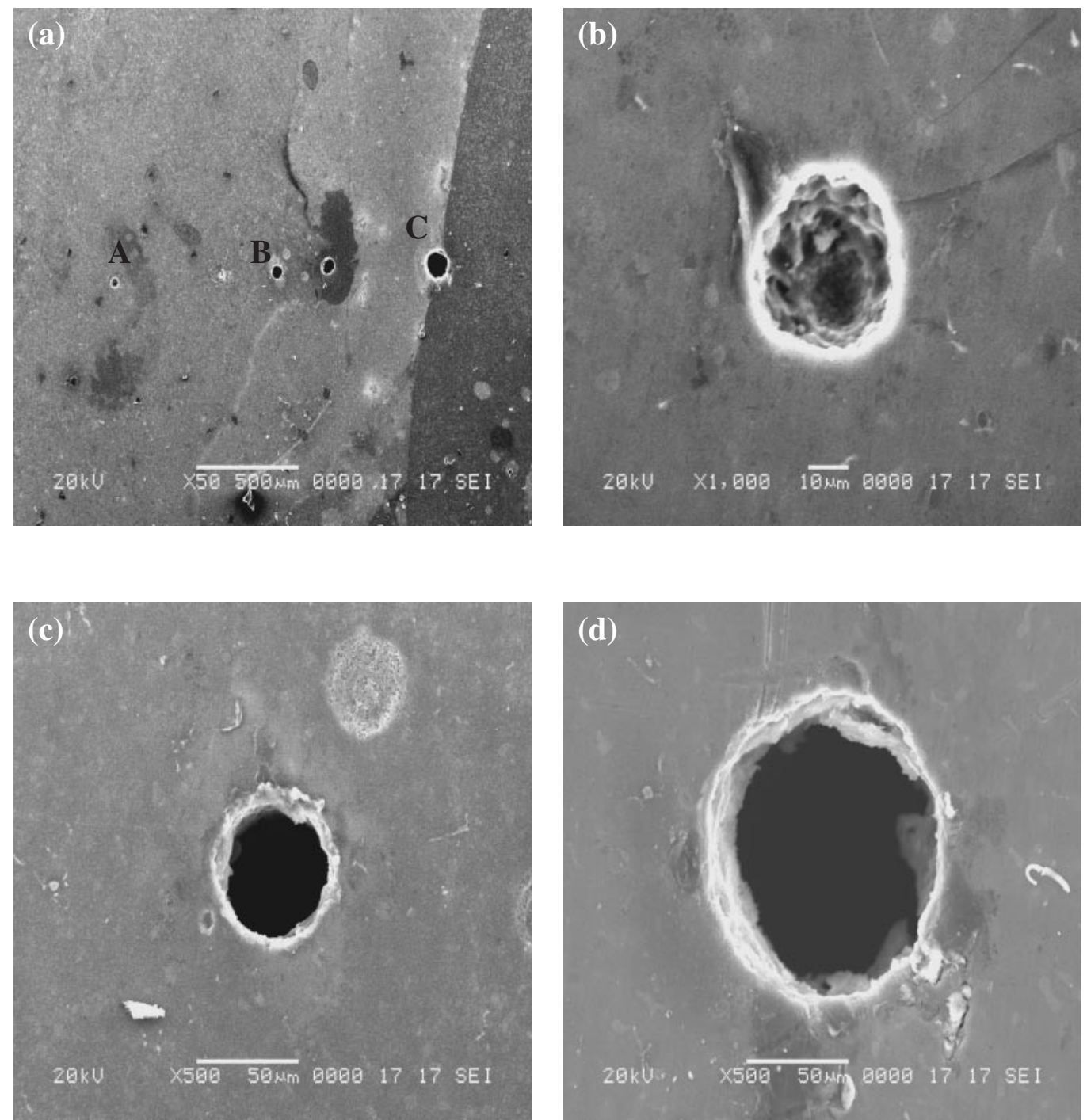

Fig. 1 Dimensions of artificial holes (Depth of artificial holes with $\varphi 50 \mu \mathrm{m}$ and $\varphi 100 \mu \mathrm{m}$ was $300 \mu \mathrm{m}$ ). (a) full scale, (b) artificial hole A with $\varphi 20 \mu \mathrm{m}$, (c) artificial B with $\varphi 50 \mu \mathrm{m}$, (d) artificial C with $\varphi 100 \mu \mathrm{m}$ in Fig. 1(a).
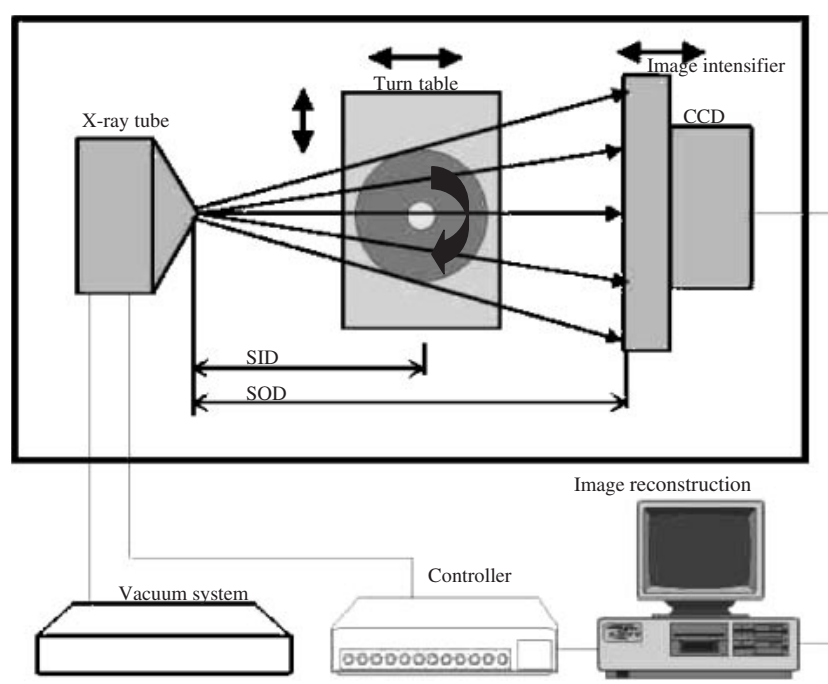

Fig. 2 Schematic diagram of the XRCT system. length, were made by casting the commercial AZ91D alloy into the Ransley mold. Ransley mold is primarily used for making the specimen of hydrogen gas analysis and can make the defect-free specimen by the directional solidification. ${ }^{6)}$ The artificial holes with 20,50 and $100 \mu \mathrm{m}$ in diameter, were made by the discharge machining as shown in Fig. 1. In this study, the dimensions of micro porosity should be measured

Table 1 Experimental condition of X-ray computed tomography.

\begin{tabular}{ll}
\hline & Characteristics \\
\hline X-ray tube & $63 \mathrm{kV}, 60 \mu \mathrm{A}$ \\
\hline Image intensifier & Input plane size: 4.5 inch \\
& Pixel size: $6.25 \mu \mathrm{m}$ \\
\hline CT processing system & Windows NT4.0 based computer \\
& Reconstruction matrix: $1024 \times 1024$ \\
& Data format: BMP gray scale \\
\hline Scan mode & Slice pitch $\times$ planes: $20 \mu \mathrm{m} \times 4$ planes \\
& Scanning time: 950 seconds $/$ slice \\
\hline Variables & Image conversion coefficient of ax $+\mathrm{b}:$ \\
& a $=1,3,5,10, \mathrm{~b}=2048$ \\
& Slice width: $10,20,30,40 \mu \mathrm{m}$ \\
\hline
\end{tabular}


in different slice position by using XRCT analysis. Therefore the artificial hole must have a sufficient depth and perpendicularity. However, as the $20 \mu \mathrm{m}$ sized hole have no sufficient depth and perpendicularity for the XRCT analysis as shown in Fig. 1(b), the 50 and the $100 \mu \mathrm{m}$ sized holes were used in this study. The average diameter was measured by the scanning electron microscope (JSM-5600, Jeol Co.) and the image analysis system (Image-pro plus, Media Cybernetics Co.).

The XRCT scanning system used in this study was a SMX225CT model manufactured by Shimadzu Corporation. This system is one of 3rd generation XRCT system with a flexible geometry type for micro tomography. Figure 2 shows a schematic diagram of XRCT scanning system. As shown in Fig. 2, the XRCT scanning system consists of an X-ray tube for generating X-ray beam, a series of detectors and the object to be investigated. Data obtained from scanning are reconstructed to cross sectional image by computer. The reconstructed image was analyzed by image analysis software. The image conversion coefficient $a$ based on the characteristics of material, changed with $1,3,5$ and 10 . The slice width changed with $10,20,30$ and $40 \mu \mathrm{m}$. Table 1 show experimental conditions of XRCT system for analyzing micro porosity in AZ 91D alloys.

The effect of slice width, image conversion coefficient $a$ on the gray level and the dimension of micro porosity in the reconstructed image derived from the XRCT analysis were investigated.

\section{Results and Discussion}

\subsection{Image conversion coefficient $a$}

The reconstructed image based on this map of X-ray linear attenuation coefficient, shows the position, size, and composition of the objects in the field of view of the image intensifier. Especially, the gray level in the reconstructed image is proportional to linear attenuation factor depended on the density of objects. ${ }^{7)}$ The image conversion coefficient $a$ is used as an important factor which is directly related with Xray linear attenuation coefficient. The collected XRCT data of X-ray linear attenuation coefficient are reconstructed to the forms of gray level through the function of image conversion coefficient $a$. The gray level of reconstructed image is proportional to the image conversion coefficient $a$ as shown in eq. (2).

$$
\text { Gray level: } a \mathrm{x}+b
$$

$a$ and $b$ : image conversion coefficient

As shown in Fig. 3, outside of specimen and three artificial holes filled with air were represented as darker gray color depending on their density. Three artificial holes of specimen were detected as two $50 \mu \mathrm{m}$ sized holes and a $100 \mu \mathrm{m}$ sized hole and we called them 50-1, 50-2 and 100. The matrix was contrasted lighter gray color depending on the density.

It is commonly more desirable to select the reconstruction parameter such as image conversion coefficient $a$ to maximize the gray level contrast for each scanned object. When the image is reconstructed by the collected XRCT data, the image conversion coefficient $a$ reflects the material characteristics on the reconstructed image and affects on the sensitivity of reconstructed image. ${ }^{7)}$ Figure 3 shows the reconstructed image variation at the different image conversion coefficients $a$. With increasing the image conversion coefficient, $a$, the roughness of reconstructed image increased. The reason is that the image conversion coefficient is based on the characteristics of material such as the density. Thus, the increasing of image conversion coefficient $a$, increased the sensitivity of reconstructed image and brought even the difference of gray level of matrix interior. We think that this difference of gray level of matrix interior is resulted from the material characteristics such as the secondary dendrite arm size, solute segregation and the grain size.

For the gray level of artificial hole at $a=1$, we investigated the variation of gray level using the line profile analysis in the reconstructed image as shown in Fig. 4(a). In Fig. 4(b), the blue line shows the gray level variation with the distance in the $100 \mu \mathrm{m}$ sized artificial hole, and the red line shows the gray level variation with the distance in the $50 \mu \mathrm{m}$ sized artificial hole. In the both cases, the gray level was not a specific value and changed with the distance.

In order to use these characteristics of gray level for dimensions of artificial hole, the gray level variation in the reconstructed image was investigated as shown in Fig. 5. As shown in Fig. 5(a), the region of air could be evidently divided from the region of matrix in the gray level variation at $a=1$. Besides only a few peaks were shown and the intensity of signal was sufficient. However, increasing of image conversion coefficient, increased the gray level width of signal, decreased the intensity and made too much peaks. Therefore, the region of air could not be distinguished from the region of matrix in the case of $a=3,5$ and 10 .

Because the gray level of reconstructed image is directly related with the density, the defining of peak in the variation of gray level is very important for analyzing the porosity or compounds inside the matrix. In this study, we used the defining of the peaks in the boundary region of air with matrix to precisely analyze dimensions of artificial holes. Figure 5(b) shows the gray level variation of reconstructed image at $a=1$. In Fig. 5(b), the small peak was in the boundary region of air with matrix. The small peak had three positions in gray level. Three positions were the start position at 121 , the top position at 122 , and the next bottom position at 123. We investigated into the variation of analyzed image at three positions of this peak in the boundary region of air with matrix, as shown in Fig. 6.

Figure 6 shows the variation of analyzed image with changing the gray level range in the defect-free specimen without artificial holes (a) and with those (b) $\sim$ (d). Yellow region in Fig. 6 indicates the detected region at the range of gray level for analysis. As Fig. 6(a) is the result of defect-free specimen without artificial holes, the porosity of matrix was not detected at the gray level $0 \sim 121$. Only the three artificial holes were detected at the gray level $0 \sim 121$ in the specimen with artificial holes in Fig. 6(b). However, besides artificial holes, the porosities were detected in the matrix at the gray level $0 \sim 122$ and $0 \sim 123$ as shown in Figs. 6(c) and (d). These porosities were not real porosity, and were resulted from matrix characteristics such as small grain and solute segregation of inter dendrite. Therefore, the gray level 121 could be the critical value which it distinguished the 

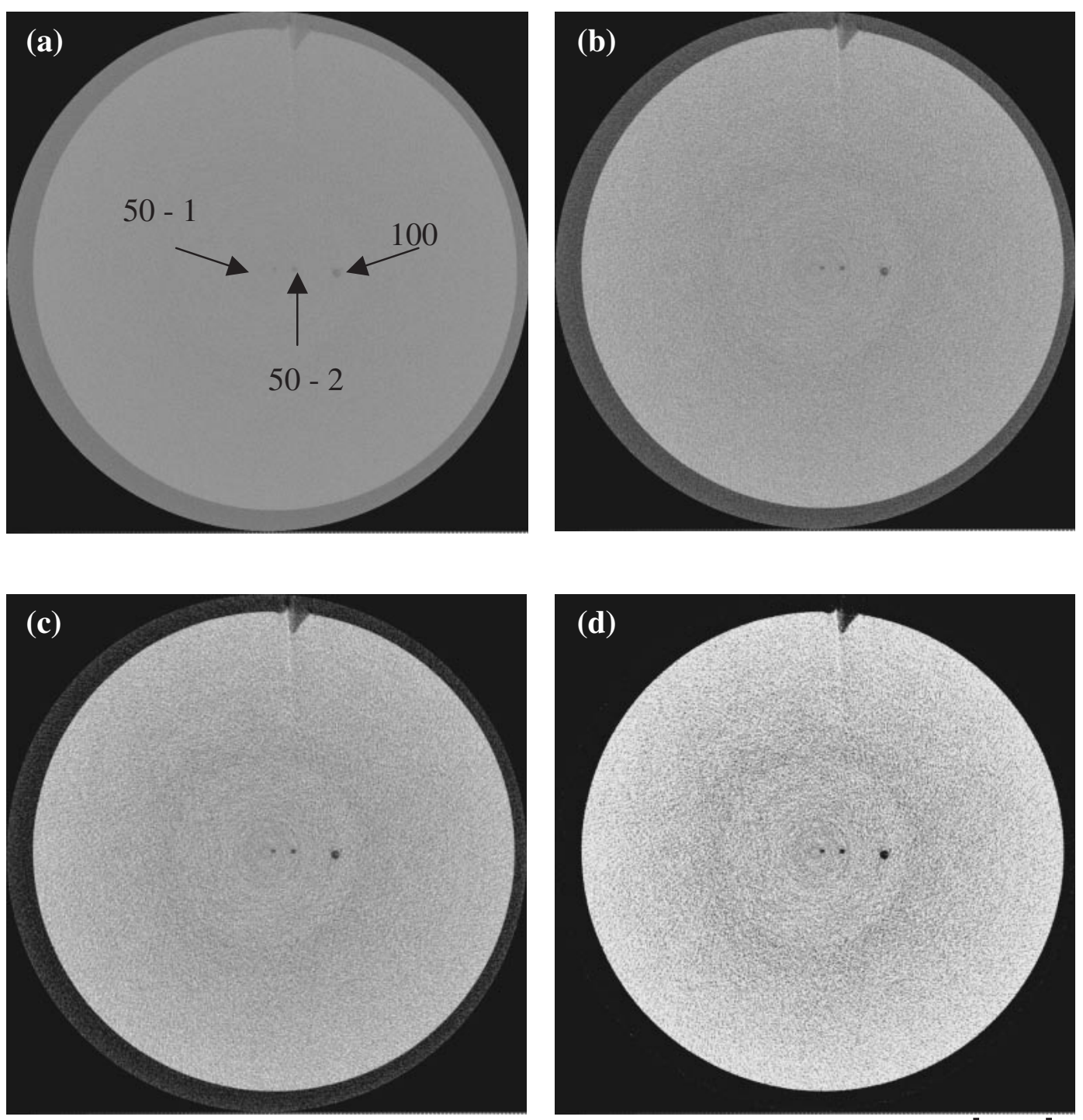

Fig. 3 Reconstructed image variation with image conversion coefficient $a$ change (slice width: $20 \mu \mathrm{m}$ ). (a) $a=1$, (b) $a=3$, (c) $a=5$, (d) $a=10$.

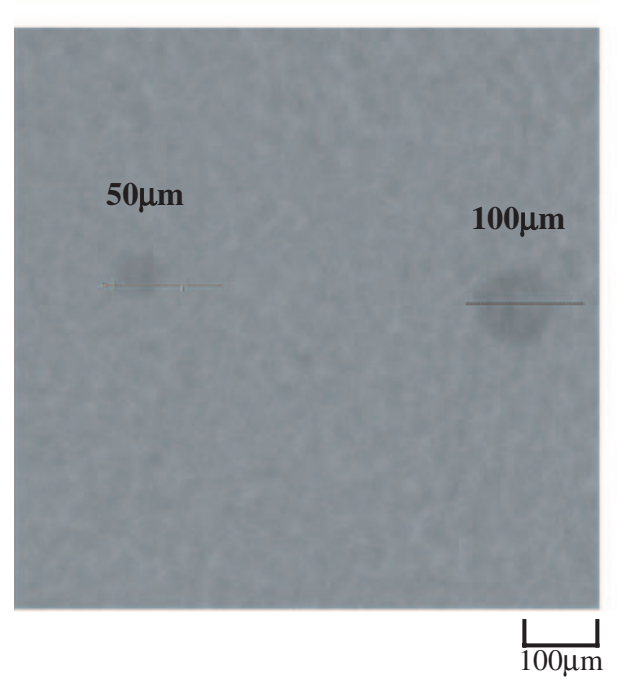

(a) XRCT image

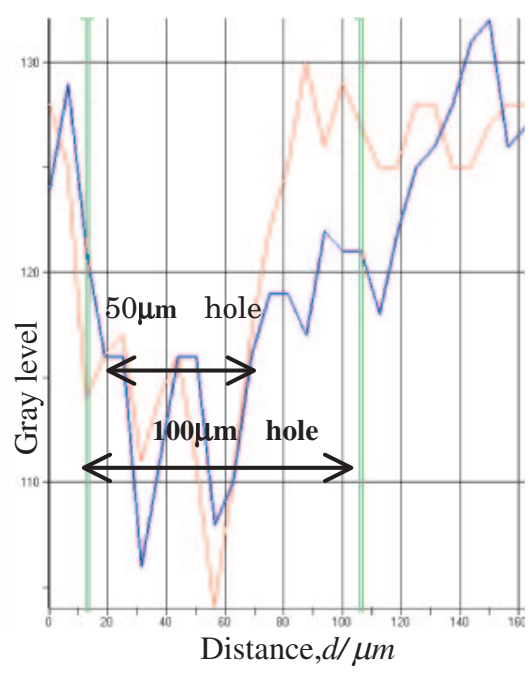

(b) Variation of gray level

Fig. 4 Line profile results of artificial holes in XRCT analysis. (a) XRCT image (b) Variation of gray level. 


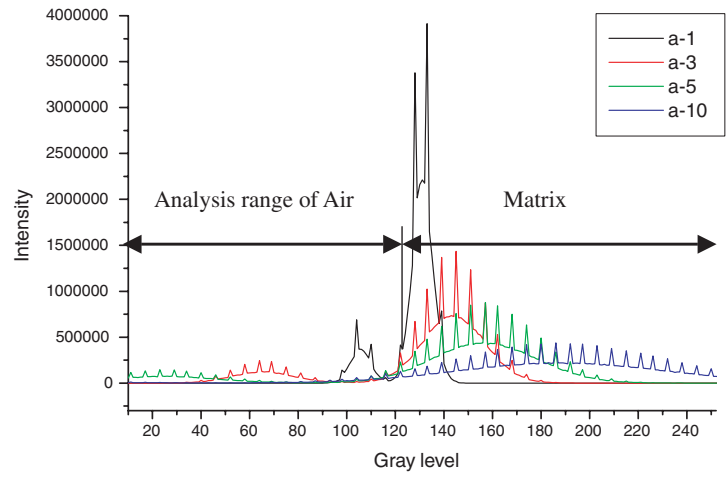

(a) Relation of image conversion coefficient $a$ change with gray level

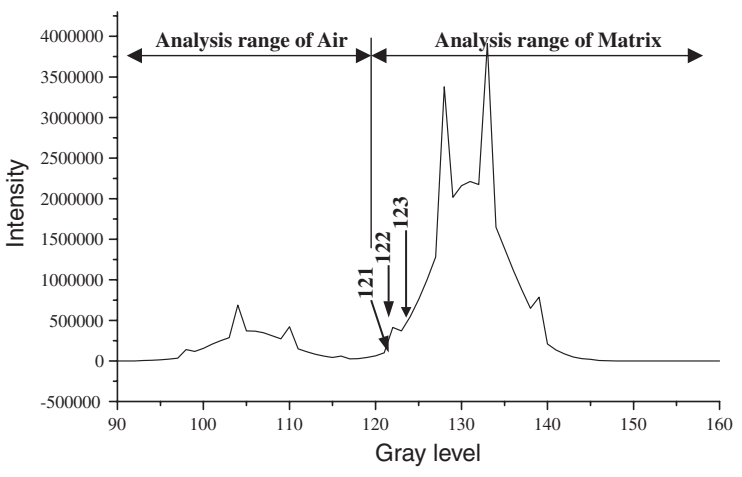

(b) Gray level variation of reconstructed image at the image conversion coefficient $a=1$

Fig. 5 Relation of gray level of reconstructed image with the image conversion coefficient $a$. (a) Relation of image conversion coefficient $a$ change with gray level (b) Gray level variation of reconstructed image at the image conversion coefficient $a=1$.
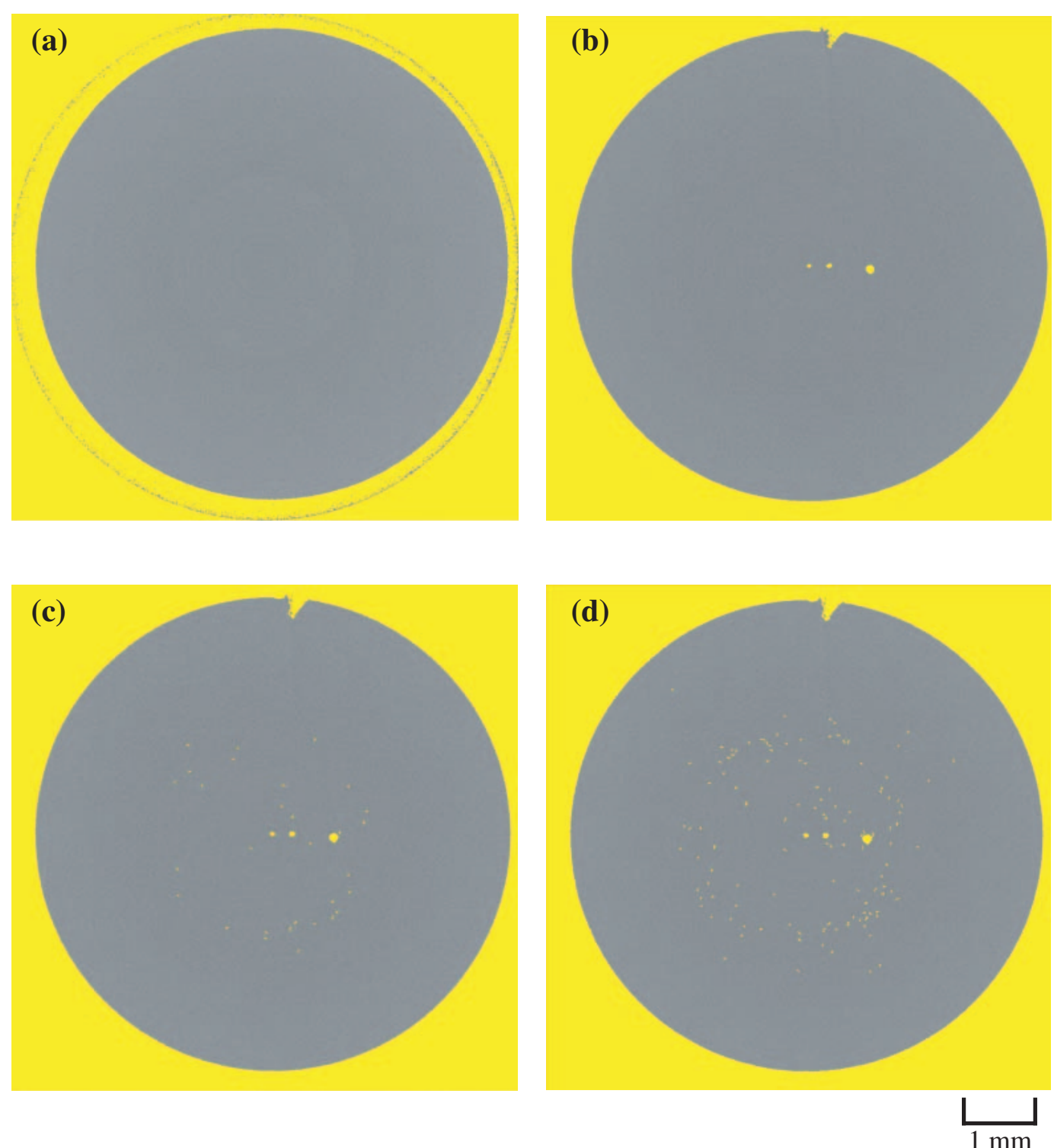

Fig. 6 Variation of analyzed image with changing the gray level range in the defect-free specimens(slice width $20 \mu \mathrm{m}, a=1$ ). (a) Range $0 \sim 121$ in defect-free specimen without artificial holes, (b) Range $0 \sim 121$ in specimen with artificial hole, (c) Range $0 \sim 122$ in specimen with artificial hole, (d) Range $0 \sim 123$ in specimen with artificial hole. 

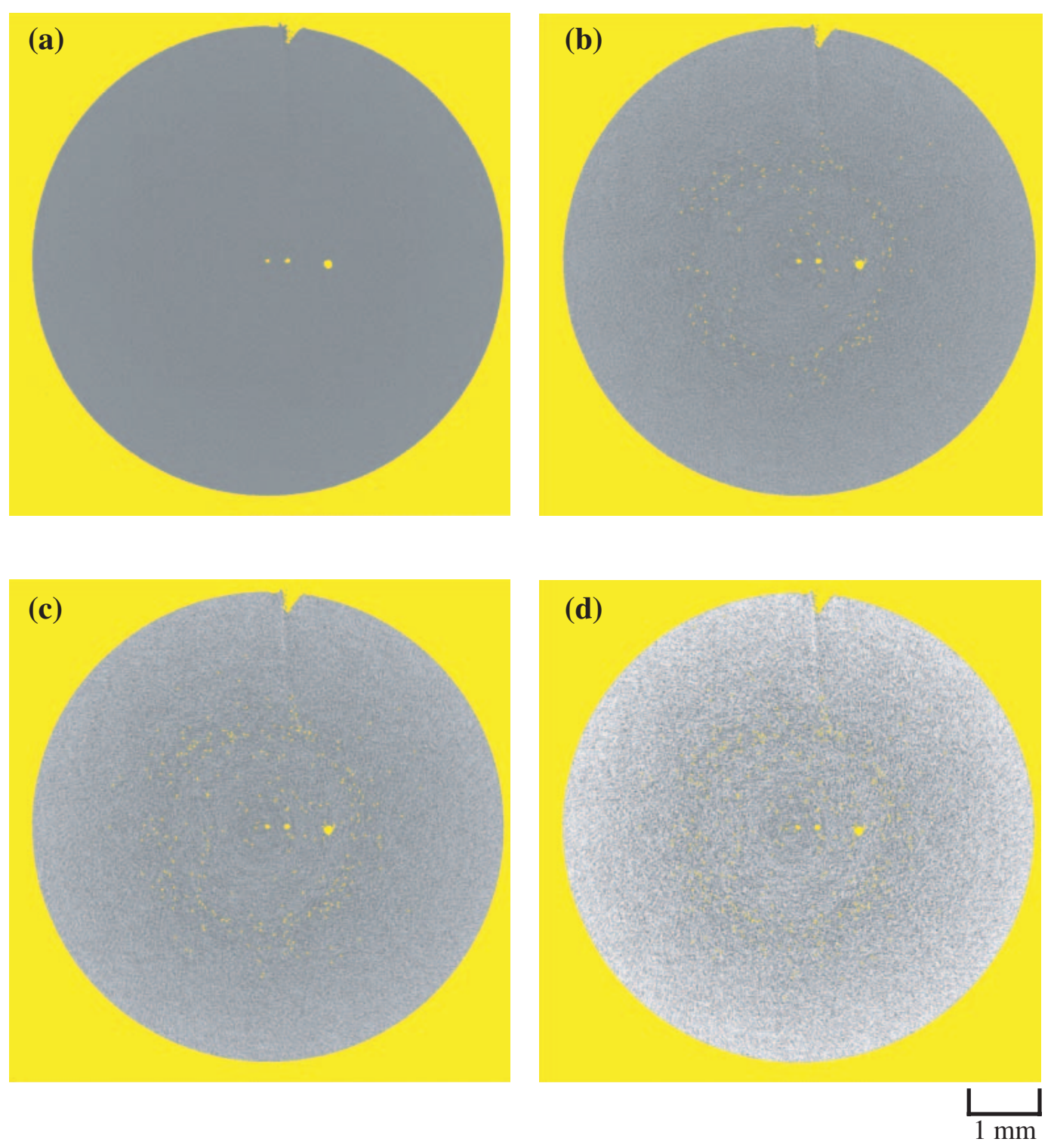

Fig. 7 Variation of analyzed image with image conversion coefficient $a$ change at the gray level $0 \sim 121$ (slice width: $20 \mu \mathrm{m}$ ). (a) $a=1$, (b) $a=3$, (c) $a=5$, (d) $a=10$.

artificial holes from the matrix and was used for measuring the dimensions of artificial hole in this case.

Figure 7 shows the variation of analysis results with changing the image conversion coefficient at the gray level $0 \sim 121$. While only the artificial holes were detected as the porosity at $a=1$, the artificial holes and the matrix were detected as the porosity at $a=3,5$ and 10 . The amounts of regions detected as the porosity, increased with increasing the image conversion coefficient $a$.

From these results, we could conclude that the optimized image conversion coefficient $a$ was 1 for the XRCT analysis of micro porosity in AZ91D alloy. It was very important to optimize the image conversion coefficient $a$ for precise analysis in lightweight metallic materials which have very fine grain size and microsegregation. Also, the optimized image conversion coefficient should be considered with the purpose of the analysis and the characteristics of material.

\subsection{Slice width}

Slice width is related with the amounts of collected XRCT signal and affects the quality of reconstructed image and resolution. In general, the collected XRCT signal was increased with the increase of slice width. This can increase the quality of reconstructed image. ${ }^{3)}$ But the excessive increase of slice width may change the shape of porosity and can erase porosity in the reconstructed image. Especially, in the case of porosity with an order of micrometer, these problems occur very frequently. Therefore, it is very sensitive and important to select the optimized slice width which it can measure the actual dimensions of micro porosity in XRCT analysis.

Figure 8(a) shows the amounts variation of regions detected as the porosity with the slice position change in the same defect-free specimen with only three artificial holes. Slice plane were four plane and slice pitch between the planes was $20 \mu \mathrm{m}$ as shown in Table 1. In the case of slice width $10 \mu \mathrm{m}$, the amounts of regions detected as the porosity, largely deviated with the slice position change from 1st plane to 4th plane. This means that even the matrix was detected as the porosity at slice width $10 \mu \mathrm{m}$. Contrast with this, the 


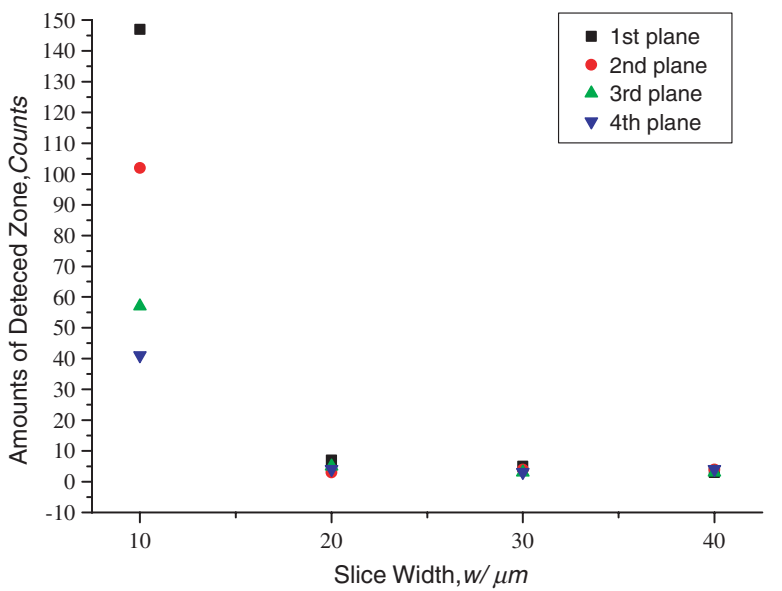

(a) Amounts variation of detected zones with analysis position change

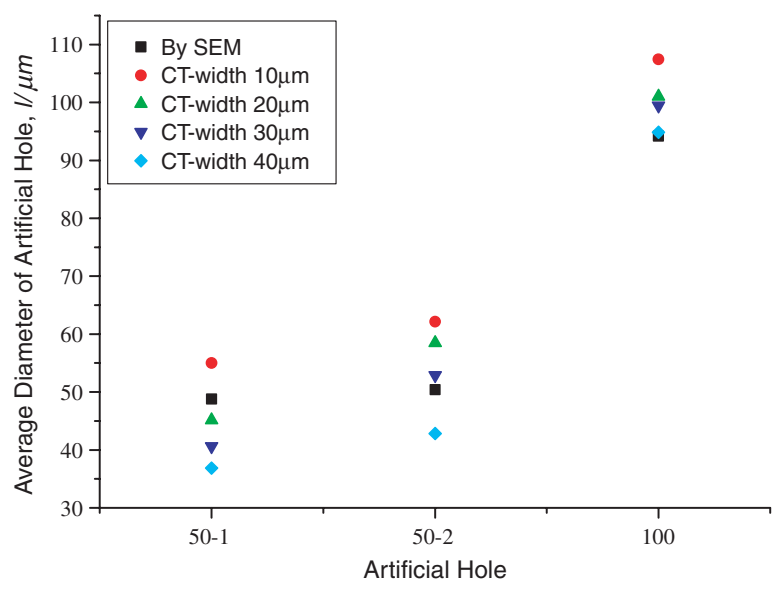

(b) Average diameter variation with slice width change

Fig. 8 Dimensions variation derived from XRCT with slice width change. (a) Amounts variation of detected zones with analysis position change, (b) Average diameter variation with slice width change.

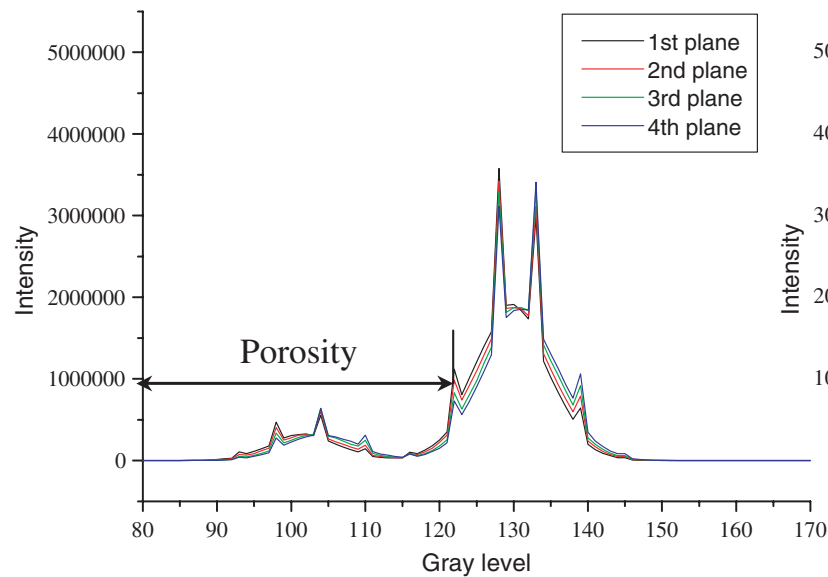

(a) slice width $10 \mu \mathrm{m}$

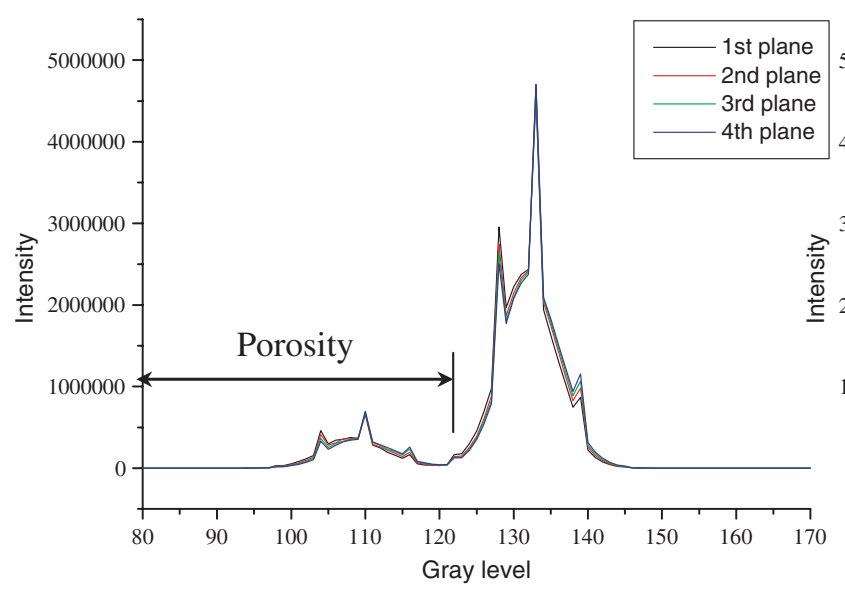

(c) slice width $30 \mu \mathrm{m}$

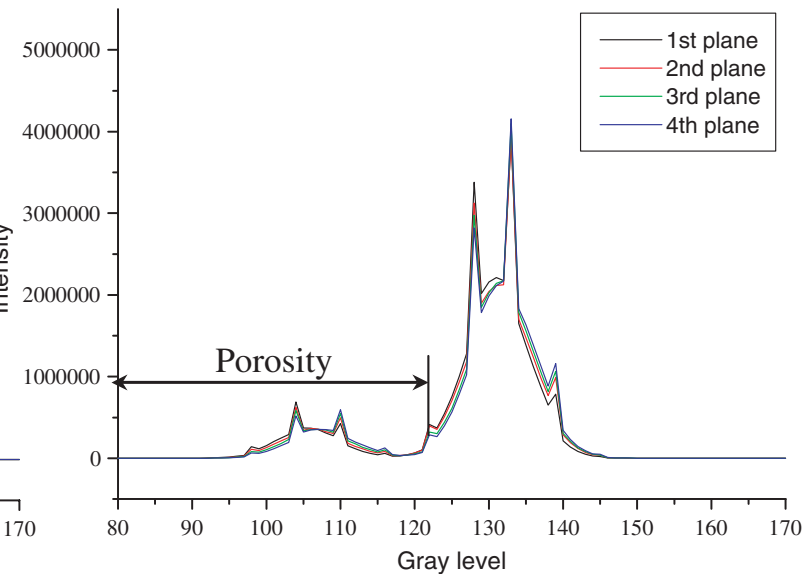

(b) slice width $20 \mu \mathrm{m}$

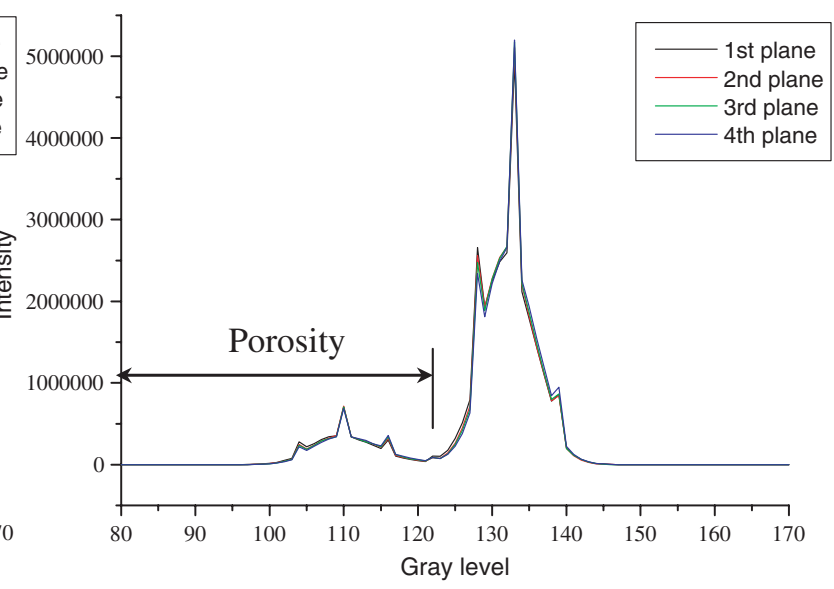

(d) slice width $40 \mu \mathrm{m}$

Fig. 9 Gray level variation with slice width change at four slice positions. (a) slice width: $10 \mu \mathrm{m}$ (b) slice width: $20 \mu \mathrm{m}$ (c) slice width: $30 \mu \mathrm{m}$ (d) slice width: $40 \mu \mathrm{m}$.

amounts of detected regions did not change with the slice position change at slice width 20,30 , and $40 \mu \mathrm{m}$.

The slice width affects not only the amounts of region detected as porosity but also their average diameter. Figure 8 (b) shows the comparison of the average diameter measured by SEM with that measured by XRCT analysis. The average diameter at slice width $10 \mu \mathrm{m}$, denoted the largest difference from that measured by SEM. However, the average diameter of the $50 \mu \mathrm{m}$ sized artificial holes at slice width 20 and $30 \mu \mathrm{m}$ had the nearest value from that measured by SEM. And in the 
case of the $100 \mu \mathrm{m}$ sized artificial holes, the average diameter at slice width $40 \mu \mathrm{m}$ had the nearest value from that measured by SEM. This means that the optimized slice width which it can measure the actual dimensions of micro porosity, depend on the size of micro porosity. If the slice width was too thin to analyze only the porosity as like the case at slice width $10 \mu \mathrm{m}$, even the matrix would be detected as the porosity and the results would include the serious error. Thus, the dimensions of micro porosity measured by XRCT were larger than actual dimension.

These results agreed with the gray level variation as shown in Fig. 9. In the case of slice width $10 \mu \mathrm{m}$, the deviation of intensity with the position was larger and the intensity was lower than those of another cases. And the deviation of intensity decreased with increasing the slice width. Therefore, the accuracy of data derived from XRCT depended on the slice width related the amounts of collected data.

\section{Conclusions}

In order to determine the actual size and distribution of the porosity with an order of micrometer in AZ91D alloy, it is especially important to use the optimized operating conditions and the proper analysis method which consider the purpose of analysis and the characteristics of material. The results are summarized as follows.

(1) The defining of peak in the variation of gray level could be used for analyzing the porosity or compounds. In the case of artificial holes in AZ91D alloy, the gray level was not a specific value and changed in the range under 121 with order of gray level.

(2) Increasing of image conversion coefficient $a$, increased the sensitivity of reconstructed image and brought even the difference of gray level of matrix. This difference gray level of matrix was resulted from the characteristics of material such as the secondary dendrite arm size, solute segregation and the grain size. The optimized image conversion coefficient was 1 for the XRCT analysis of micro porosity in AZ91D alloy. The optimized image conversion coefficient should be considered with the purpose of the analysis and the characteristics of material.

(3) The optimized slice width which it can measure the actual dimensions of micro porosity, depends on the size of micro porosity. If the slice width was too thin to analyze only the porosity as like the case at slice width $10 \mu \mathrm{m}$, even the matrix would be detected as the porosity and the results would include the serious error. The optimized slice width was above $20 \mu \mathrm{m}$ for XRCT analysis of micro porosity with size range $50 \sim 100 \mu \mathrm{m}$ in AZ91 alloy. The accuracy of data derived from XRCT depends on the slice width related the amounts of collected data.

\section{Acknowledgements}

We are very grateful to Prof. Hideki Yasuda in Osaka University and Dr. Rudi Suhradi Rachmat in AIST for their useful discussions about X-ray Computed Tomography analysis.

\section{REFERENCES}

1) P. M. Mummery, B. Derby and P. Anderson: J. Microsc. 177 (1995) 399406.

2) S. R. Stock: Int. Mater. Rev. 44 (1999) 141-164.

3) R. W. Hamilton, M. F. Forster, R. J. Dashwood and P. D. Lee: Scr. Materialia 46 (2002) 25-29.

4) C. F. Buynak and R. H. Bossi: Nucl. Instrand Meth. in Phys. Res. B99 (1995) 772-774.

5) O. G. Duliu: Earth Science Rev. 48 (1999) 265-281.

6) C. E. Ransley and H. Neufled: J. Inst. Metals 74 (1948) 599.

7) D. H. Phillips and J. J. Lannutti: NDT \& E Int. (30) (1997) 339-350.

8) R. A. Ketcham and W. D. Carlson: Computers \& Geosciences 27 (2001) 381-400. 